\title{
Nicorandil Protects the Heart from Ischemia/Reperfusion Injury by Attenuating Endoplasmic Reticulum Response-induced Apoptosis Through PI3K/Akt Signaling Pathway
}

\author{
Hui Wu ${ }^{a}$ Ming Ye $e^{a}$ Jun Yang ${ }^{a}$ Jiawang Ding ${ }^{b}$ Jian Yang ${ }^{b}$ Wusong Dong \\ Xinan Wang ${ }^{b}$ \\ Institute of Cardiovascular Diseases, China Three Gorges University, Yichang, Hubei, China, \\ bDepartment of Cardiology, Yichang Central People's Hospital, Yichang, Hubei, China
}

\section{Key Words}

Nicorandil • Heart $・$ Ischemia/Reperfusion • Endoplasmic Reticulum • Apoptosis • PI3K/Akt

\begin{abstract}
Background/Aims: As a vasodilatory drug used to treat angina, nicorandil has been shown to induce an infarct-limiting effect in various experimental animal models of myocardial ischemiareperfusion (IR). There are multiple mechanisms causing the IR injury, among which, the endoplasmic reticulum (ER) stress and ER stress-initiated apoptosis are implicated to play an important role. However, whether ER stress is involved in nicorandil-induced cardioprotection is unknown. Methods: Post-ischemic functional recovery, lactate dehydrogenase (LDH) release and infarct size in perfused rat hearts subjected to global no-flow I/R were measured to analysis the effect of nicorandil and ER stress inducer of tunicamycin as well as phosphatidylinositol 3-kinase (PI3K) inhibitor of wortmannin on the I/R hearts. The I/R hearts tissue were harvested to evaluate apoptosis ratio with TUNEL assay and protein expression with western blot. Results: We showed that nicorandil ameliorated postischemic contractile recovery, as well as significantly reduced myocardial infarct size at a dose-dependent manner. Furthermore, nicorandil treatment inhibited the IR-induced apoptosis and ER stress. The beneficial effects of nicorandil were blocked by ER stress inducer, tunicamycin and specific phosphatidylinositol 3-kinase (PI3K) inhibitor, wortmannin. Concolusion: We conclude that the cardioprotection of nicorandil was at least in part mediated via inhibition of ER stress-induced apoptotic cell death through PI3K/Akt pathway.
\end{abstract}

H. Wu and M. Ye contributed equally to this work.

Jun Yang

KARGER 125 


\section{Cellular Physiology Cell Physiol Biochem 2015;35:2320-2332 \begin{tabular}{l|l} 
and Biochemistry Published online: April 15, 2015 & $\begin{array}{l}\text { C 2015 S. Karger AG, Basel } \\
\text { www.karger.com/cpb }\end{array}$ \\
\hline
\end{tabular} \\ Wu et al.: Nicorandil Inhibit ER Response-Induced Apoptosis During I/R Injury in the Heart}

\section{Introduction}

Ischemia and reperfusion (IR) is a disorders characterized by an initial reductions in organ-specific blood supply followed by the subsequent restoration of perfusion and detrimental consequences. IR-elicited tissue injury are implicated in morbidity and mortality in a wide range of pathologies, including myocardial infarction $[1,2]$. Acute myocardial infarction (AMI) is responsible for the death of millions of persons worldwide each year [3].

The endoplasmic reticulum (ER) is an organelle that has an essential role in the folding and transport of secreted proteins as well as $\mathrm{Ca}^{2+}$ storage. Any disturbance of its microenvironment, or disruption of protein folding or transport within the ER, triggers the unfolded protein response (UPR) and ER-associated protein degradation (ERAD) [4]. Both pathways are intended to adapt to the changing environment and restore normal ER function. However, ER stress will occur and trigger pro-apoptotic pathways to initiate cell death when adaption fails [5]. Glucose-regulated protein 78 (GRP78), an UPR target gene, is one of the best-characterized ER chaperone proteins. Upon ER stress, sequestration of GRP78 by unfolded proteins will activate the ER stress sensors [6-10]. However, prolonged and/or excess ER stress will eventually result in significant apoptosis and could be a main contributor to myocardium IR injury [11-14].

Recently, numerous evidence have strongly indicated that ER stress plays a crucial role during IR injury $[2,15,16]$. UPR is initially activated in the myocardium after IR, as demonstrated by the upregulation of ER-targeted cytoprotective Grp78 and Grp94 [11, 17, 18]. Progressively, the ER stress response switched from UPR and cytoprotective response to a pro-apoptotic response in a later time, as evidenced by the upregulation of the proapoptotic protein C/EBP homologous protein (CHOP) and pro-caspase-12 during IR [19, 20] Increased CHOP expression further contributed towards cardiomyocytes death [21, 22], which is the main detrimental effect of myocardial ischemia-reperfusion injury $[16,19]$.

Nicorandil, an adenosine triphosphate (ATP)-sensitive potassium channel activator and nitrate agonist, have been shown to be effective for the reduction of myocardial damage after AMI in basic and clinical studies [23-25]. In IR models, nicorandil prevents oxidative stressinduced apoptosis and reduces the size of infarction [26,27]. Ishii et al found that nicorandil has a preconditioning-like cardioprotective effect against ischemic injury in myocardial infarction patients [25]. Studies in cultured cardiac myocytes suggested its antiapoptotic effect is at least partially mediated by selective activation of mitochondrial $\mathrm{K}_{\text {ATP }}$ channels [26]. However, whether nicorandil plays a role in IR-induced ER stress remains unclear.

Given that ER stress can trigger cell apoptosis, which functions as one of the leading causes of IR injury, we speculated that there might be an association between the cardiaoprotection role of nicorandil against ER stress in IR animal model. We thus evaluated the possible molecular mechansism of nicorandil in modulating ER stress. Here, we demonstrated that nicorandil improves the cardiac contractility recovery in isolated IR rat heart, and nicorandil also efficiently prevents ER stress-initiated apoptosis in a dosedependent manner. Furthermore, we showed that the attenuation of IR-induced ER stress by nicorandil is dependent on phosphatidylinositol 3-kinase (PI3K)/Akt pathway. Our study provides a novel mechanistic insight of cardioprotection role of nicorandil in IR and could be used as an important aspect to assess the therapeutic opportunities of nicorandil in other related diseases.

\section{Materials and Methods}

Animals

Eight week-old male Sprague-Dawley rats, weighing between $250 \mathrm{~g}$ and $280 \mathrm{~g}$ were obtained from the Experiment Animal Center of WuHan University. The animals were allowed to move freely, with water and food continually available, and were kept at room temperature. All animal experiments were performed in accordance with the guidelines for laboratory animal care of Medical College of WuHan University, Hubei, China. 


\section{Cellular Physiology Cell Physiol Biochem 2015;35:2320-2332 \begin{tabular}{ll|l} 
and Biochemistry $10.1159 / 000374035$ & Published online: April 15, 2015 & $\begin{array}{l}\text { C 2015 S. Karger AG, Basel } \\
\text { www.karger.com/cpb }\end{array}$ \\
\hline
\end{tabular} \\ Wu et al.: Nicorandil Inhibit ER Response-Induced Apoptosis During I/R Injury in the Heart}

\section{Chemicals and antibodies}

Tunicamycin (TM), an inhibitor of protein glycosylation which induces ER stress and apoptosis; and PI3K inhibitor, Wortmannin were from Sigma-Aldrich. Anti-Bax, anti-Bcl2, anti-phospho-Akt (Ser-473), and anti-Akt antibodys were from Cell Signaling Technology (Danvers, MA, USA). Anti-GAPDH antibody was from Sigma-Aldrich. Anti-GADD153/CHOP antibody was from Abcam (Cambridge, UK).

\section{Heart perfusions and Experimental protocol}

Male Sprague-Dawley rats were anesthetized with an intraperitoneal injection of pentobarbital sodium $(50 \mathrm{mg} / \mathrm{kg})$. The hearts were rapidly removed and immediately arrested in ice-cold buffered KrebsHenseleit (KHB) buffer containing (in mmol/L) $118 \mathrm{NaCl}, 4.7 \mathrm{KCl}, 1.2 \mathrm{KH}_{2} \mathrm{PO}_{4}, 1.2 \mathrm{MgSO}_{4}, 10$ glucose, 2.5 $\mathrm{CaCl}_{2}$, and $2.5 \mathrm{NaHCO}_{3}(\mathrm{pH}=7.4)$. Excess tissue was removed, the aorta was rapidly cannulated. The hearts were mounted in the Langendorff apparatus using $\mathrm{KHB}$ equilibrated with $95 \% \mathrm{O}_{2} / 5 \% \mathrm{CO}_{2}$ at a constant pressure of about $75 \mathrm{mmHg}$ at $37^{\circ} \mathrm{C}$. A water-filled latex balloon, connected to a pressure transducer (SP844, Memscap, Norway) and coupled to a high performance data acquisition system (PowerLab 8/30, Chart Pro software-MLS250) for continuous recording, was inserted into the left ventricle (LV) through an incision in the left atrium and inflated to set an end diastolic pressure of 5-10 mm Hg during initial equilibration. Left ventricular systolic function was assessed by recording the left-ventricular developed pressure (LVDP), which was calculated from the difference between left-ventricular systolic pressure (LVSP) and left-ventricle end-diastolic pressure (LVEDP). The LVDP, LVEDP, the maximal rate of pressure development $(+\mathrm{dP} / \mathrm{dt})$, and the maximal rate of pressure decay $(-\mathrm{dP} / \mathrm{dt})$ were continuously recorded. Heart rate and coronary flow rate were monitored simultaneously.

All hearts underwent 25-min of stabilization during which nicorandil or/and TM were present for comparison of effects upon followed IR. The 30-min of global isothermic ischemia was induced by halting perfusion and immersing the heart in perfusion buffer at $37^{\circ} \mathrm{C}$, and then 120 -min of reperfusion (Fig. 1). Rats were randomly assigned to one of the following groups: non-IR control groups; IR groups; IR groups with different dose of nicorandil; IR groups with nicorandil and TM; IR group with TM and nicorandil.

\section{Triphenyl Tetrazolium Chloride Staining}

Hearts exposed to global ischemia and reperfusion were frozen at $-80^{\circ} \mathrm{C}$. The heart was sliced into 2-mm cross-sectional pieces and sections were incubated for $30 \mathrm{~min}$ with 1\% 2,3,5-triphenyl tetrazolium chloride (TTC) stain in KHB at $37{ }^{\circ} \mathrm{C}$ to distinguish between viable (red) and nonviable (white) tissue. Sections were placed on glass slides and scanned on a Canon scanner. Infarct size was quantified using NIHImage J software, and expressed as proportion Infarct zone in whole LV area.

Terminal deoxynucleotidyl transferase-mediated 2'-deoxyuridine 5'-triphosphate nick-end labeling staining (TUNEL)

Hearts fixed in $10 \%$ formalin were embedded in paraffin and sectioned into 4 - $\mu$ m-thick slices. To detect apoptotic cells, TUNEL labeling was performed using an In Situ Apoptosis Detection kit (Roche Diagnostics $\mathrm{GmbH}$, Basel, Switzerland). To analyze the number of apoptotic cells in the infracted hearts, digital images were acquired at $\times 400$ magnification, and 5 random fields from each sample were quantified. We counted the number of TUNEL-positive cardiomyocytes and hematoxylin-stained nuclei in the entire section of each sample.

Preparation of whole cell extracts from myocardium and Western blotting analysis

Upon completion of the experimental period, the myocardium were lysed in ice-cold radioimmunoprecipitation assay (RIPA) lysis buffer containing $1 \mathrm{mmol} / \mathrm{L}$ phenylmethylsulfonyl fluoride (PMSF), $1 \mu \mathrm{g} \mathrm{ml} / \mathrm{m} 1$ leupeptin, $1 \mu \mathrm{g} / \mathrm{ml}$ aprotinin and $1 \mu \mathrm{g} / \mathrm{ml}$ pepstatin at $4^{\circ} \mathrm{C}$ for $15 \mathrm{~min}$. The homogenate was centrifuged. and the supernatant was collected. The protein concentration was determined using the bicinchoninic acid (BCA) protein assay kit according to the manufacturer's protocol (Bio-Rad). The detergent soluble supernatant was frozen with liquid $\mathrm{N}^{2}$, and stored at $-70^{\circ} \mathrm{C}$.

For western blot assay, the supernatant was mixed with Laemmli buffer and heated for 5 minutes at $95^{\circ} \mathrm{C}$. Soluble extracts (50 ug) were loaded in each lane and separated by SDS-polyacrylamide gel electrophoresis (PAGE). After electrophoresis, proteins were electrophoretically transferred to a polyvinylidene difluoride (PVDF) filter membrane (0.45 mm, Amersham). The membrane was blocked in Tris-buffered saline 


\section{Cellular Physiology Cell Physiol Biochem 2015;35:2320-2332 \begin{tabular}{ll|l} 
and Biochemistry $10.1159 / 000374035$ & Published online: April 15, 2015 & $\begin{array}{l}\text { C 2015 S. Karger AG, Basel } \\
\text { www.karger.com/cpb }\end{array}$ \\
\hline
\end{tabular} \\ Wu et al.: Nicorandil Inhibit ER Response-Induced Apoptosis During I/R Injury in the Heart}

tween-20 (TBST) with 5\% non-fat milk and incubated overnight with the corresponding primary antibodies at $4^{\circ} \mathrm{C}$. Then, the membrane was washed and incubated for $1 \mathrm{~h}$ with secondary antibody (horseradish peroxidase-conjugated antirabbit $\lg G$ ) diluted with TBST (1: 2000). The signals of detected proteins were visualized by an enhanced chemiluminescence reaction (ECL) system (Millipore, Billerica, Massachusetts, USA). The staining was quantified by scanning the films and the band density was determined with ImagePro software.

Measurement of lactate dehydrogenase $(\mathrm{LDH})$

The level of lactate dehydrogenase (LDH), an indicator of myocardial tissue injury, was measured in coronary effluent by a standard method. The activity of the enzyme was assayed in a coupled-enzyme spectrophotometric method (Perkin-Elmer, Norwalk, Connecticut, Lambda 2) by using a Cytotoxicity Detection Kit (LDH, Roche). The activity of the enzyme was normalized against the wet weight of the heart and CF rate, and expressed as unit/min/g wet weight.

Statistical analysis

Data are expressed as means \pm standard deviation (SD). Statistical significance was determined using ANOVA for multiple comparisons. Significant differences between the two mean values were estimated using Student's t-test. $\mathrm{P}<0.05$ was considered statistically significant. All statistical analyses were performed using standard software package SPSS version 12.0.

\section{Results}

Effect of nicorandil on left ventricular function during IR

To assess the cardioprotection function of nicorandil in rat heart IR, we examined the recovery of cardiac contractile function in Langendorff-perfused rat hearts (Fig. 1). During the preischemic phase, nicorandil did not affect the LVDP, LVEDP, $+\mathrm{dP} / \mathrm{dt}$, and $-\mathrm{dP} /$ dt (data not shown). However, in the reperfusion period, nicorandil significantly improved the postischemic recovery of left ventricle developed pressure (LVDP), LV end-diastolic pressure (LVEDP), and maximum rates of pressure development or decay over time $( \pm \mathrm{dp} /$ dt max), compared to the control (Fig. 2A-D). Importantly, as we increased the concentration of nicorandil between the range of $30-100 \mu \mathrm{M}$, the heart function recovery was even more dramatic, indicating that nicorandil function in protecting IR in a dose-dependent manner.

Cardiac infarct size was also measured after nicorandil treatment by Triphenyl Tetrazolium Chloride (TTC) staining. As shown in Figure 2E, the infarct size was greatly reduced in the nicorandil preconditioning group, consistent with the above observation. The leakage of lactate dehydrogenase (LDH) is an indicator of necrotic damage induced by IR [28]. We then assessed the cardioprection role of nicrorandil by examinng the leakage of LDH. As shown in Figure 2F, the level of LDH was markedly increased after IR, wheras nicorandil preconditioning significantly reduced the IR-induced release of LDH in rat hearts.

Taken together, treatment with nicorandil improves the contractile function recovery and reduces the infarction of the rat IR heart, providing a cardioprotection role against IRinduced contratile dysfunction.

Concentration-dependent responses of anti-apoptotic function of Nicorandil in IR hearts

IR injury-induced loss of cardiac cells have been long considered to be due to the necrotic cell death, but studies over the past few decades have found that cardiomyocytes loss is mainly attributed to cell apoptosis after myocardial infarction [29]. To understand why nicorandil improve the recovery of myocardial function, we examined the consequence of nicorandil treatment on cell apoptosis during IR. In deed, IR induced cell apoptosis, as evidenced by TUNEL staining in myocardium cells (Fig. 3A), whereas nicorandil preconditioning treatment reduced the TUNEL-positive cells near to the control level.

It's well known that the B cell lymphoma 2 (BCL2) family proteins are involved in the regulation of ER stress-induced cell death [30]. The Bcl2 gene family can exert their pro- or 
Fig. 1. Experimental protocol for the Langendorff perfused rat heart. After stabilization, rat hearts were perfused for 25 additional minutes (Baseline) and then subjected to $30 \mathrm{~min}$ of no-flow global ischemia followed by $120 \mathrm{~min}$ of reperfusion. Arrows indicate time points of interventions. Nic, nicorandil; TM, Tunicamycin; WM, Wortmannin.

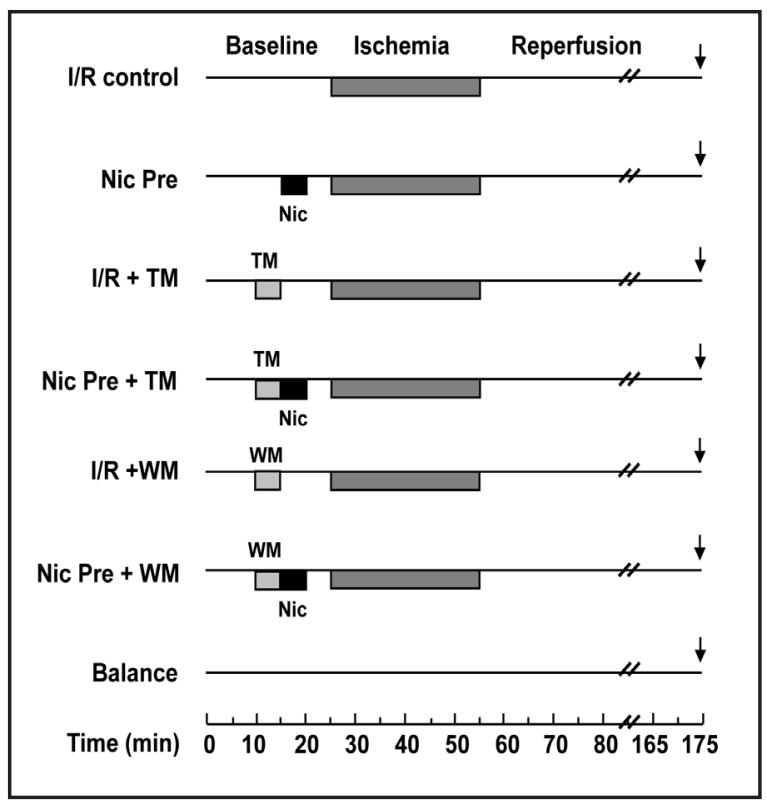

Fig. 2. Effects of nicorandil on IR-mediated myocardium injury. A to D, cadiopretection effect of nicorandil was assessed by measuring hemodynamic changes in the IR hearts. LVDP, left ventricular developed pressure; LVEDP, left ventricular end-diastolic pressure; +dP/ $\mathrm{dt}$, maximal rate of $\mathrm{LV}$ pressure development; - $\mathrm{dP} / \mathrm{dt}$, maximal rate of LV pressure decline; $\mathrm{E}$, Effects of nicorandil on myocardial infarct size. Representative heart slices with TTC staining are shown in panel $\mathrm{E}$ with quantification shown in the bar graph; F, Effects of nicorandil on the myocardial content of $\mathrm{LDH}$ in the left ventricle of rats. Nic PC: nicorandil preconditioning. Nicorandil (Nic, 30, 100, 300 $\mathrm{mM}$ ) was added before ischemia for 5 min with a 5-min washout before ischemia. $n=4-6$ in each group. Values are the Mean \pm S.E.M. ${ }^{*} \mathrm{P}<0.05,{ }^{* *} \mathrm{P}<0.01$, $* * * \mathrm{P}<0.001$ compared to balance group; \#P $<0.05$, \#\# $<0.01$ compared to IR group.

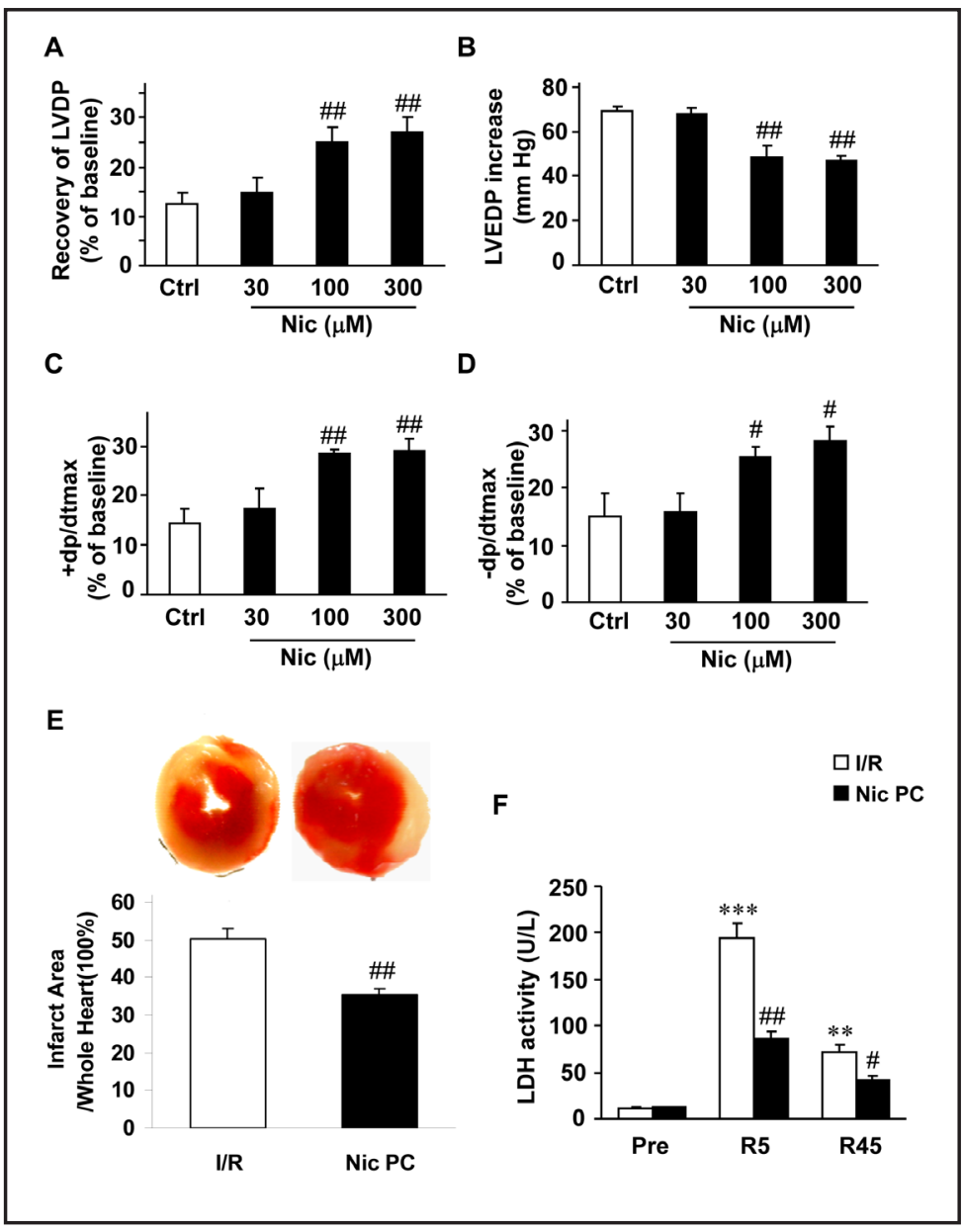

anti-apoptotic effect in response ER stress [31]. Among them, Bcl2 plays a anti-apoptotic role, while BAX is a pro-apoptotic protein. We next explored whether nicorandil can alter the BCL2 family members expression in the IR hearts. As expected, we found Bcl2 was deceased and BAX was elevated in IR hearts compared to the balance group. On the contrary, nicorandil 
Fig. 3. Effects of nicorandil on IR-mediated apoptosis. (A) Representative TUNEL staining is shown and quantitative analysis (percentage of apoptotic cells versus total) is shown in histogram. (B) Representative of western blot analysis of apoptotic factors Bcl2 and BAX. GAPDH (lower lanes) was used to demonstrate equal protein loading. Nic PC: nicorandil preconditioning. Nicorandil (Nic, 30, 100, $300 \mathrm{mM}$ ) was added before ischemia for 5 min with a 5-min washout before ischemia. $\mathrm{n}=3$ in each group. Values are the Mean \pm S.E.M . $* * * \mathrm{P}<0.001$ compared to balance group; \#P $<0.05$, \#\#P $<0.01$ compared to IR group. Black arrows indicate the TUNEL-positive cells.

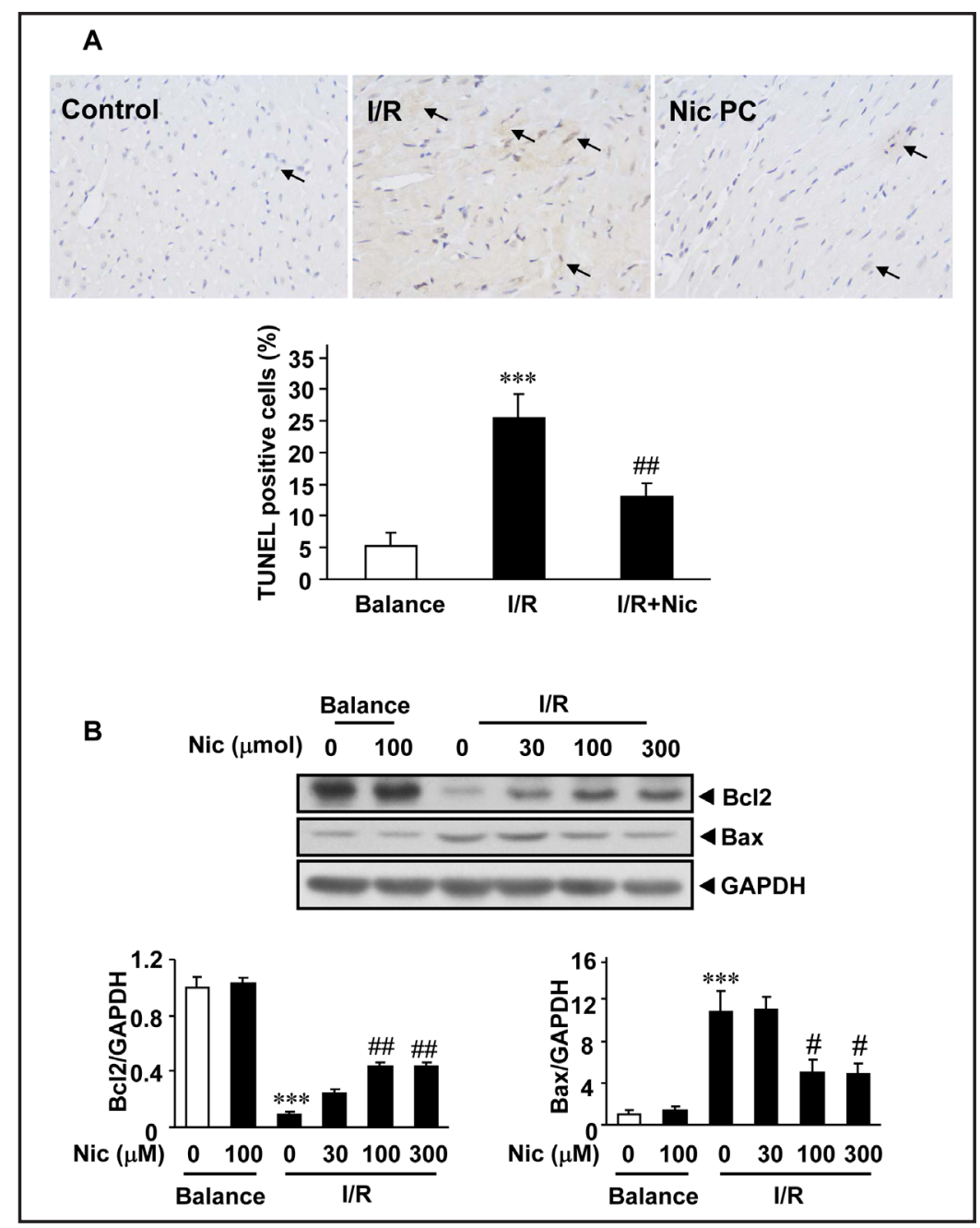

treatment suppressed the increase of BAX level and meanwhile enhanced the expression of the anti-apoptotic protein BCL2 in a dose-dependent manner (Fig. 3B), indicating that nicorandil promotes cell survival and protects the IR heart from cell apoptosis.

Concentration-dependent effects of nicorandil on IR-induced ER stress

IR injury affects the integrity of the ER and promotes ER stress [16, 19], thus we want to investigate how ER stress was involved in nicorandil treatment. Isolated simulated IR hearts were pretreated with or without nicorandil, and tissue extracts were analyzed for the levels of ER stress markers. In line with the TUNEL staining assay, ER stress related proteins exhibited a similar pattern of changes. As shown in Figure 4, the pro-apoptotic transcription factor CHOP and ER-targeted cytoprotective chaperones glucose-regulated proteins 78 (Grp78) were upregulated in IR heart. When the IR hearts were treated with nicorandil, both CHOP and GRP78 level were significantly attenuated. Importantly, nicorandil did not change the expression of CHOP and Grp78 in baseline condition. Therefore, nicorandil appears to inhibit IR-induced ER stress.

We then examined whether ER stress is functionally important for the cardioprotection role of nicorandil. We enhanced ER stress by adding $2.5 \mu \mathrm{g} / \mathrm{ml}$ tunicamycin, the ER stressinducing agent, to the IR hearts with or without nicorandil treatment. We found that tunicamycin resulted in a restoration of the level of $\mathrm{CHOP}$ and Grp78 in the nicorandil treament group (Fig. 5A). The protective effects of $100 \mu \mathrm{M}$ nicorandil on the contractile function of LVDP, $+d t / d t$ and $-d p / d t$ levels against IR were abolished by the exposure to tunicamycin 
Fig. 4. Effects of nicorandil on ER stress induded by IR. (A-C) Representative immunoblots (A) and averaged densitometric results of ER stress related protein markers including GRP78 (B) and CHOP (C). Nic PC: nicorandil preconditioning. Nicorandil (Nic, 30, 100, $300 \mathrm{mM}$ ) was added before ischemia for 5 min with a 5-min washout before ischemia. $n=4$ each. Values are the Mean \pm S.E.M. GAPDH was used as a loading control. ${ }^{*} \mathrm{P}<0.05$, ${ }^{* *} \mathrm{P}<$ $0.01,{ }^{* * *} \mathrm{P}<0.001$ compared to balance group. $\mathrm{AP}<0.05$, \#\#P $<0.01$ compared to IR group.

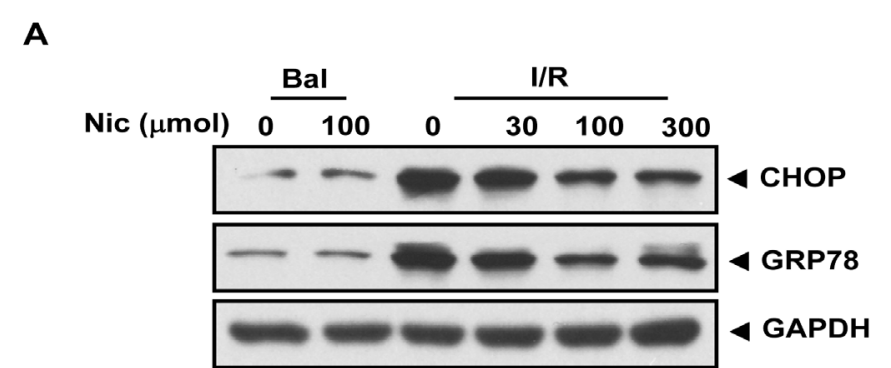

B

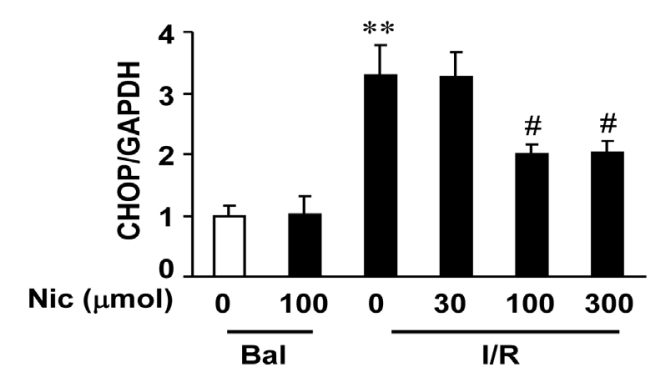

C

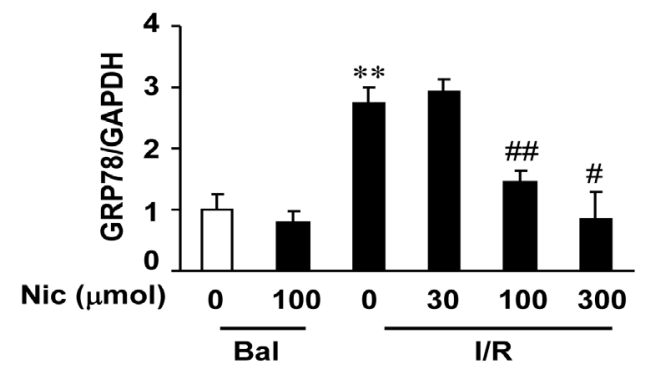

(Fig. 5B, D, E). The LVEDP was also reverted to that in the IR group (Fig. 5C). Furthermore, the infarct size was significant increased after treatment of ER stress inducer tunicamycin (Fig. 7). Worthnotely, tunicamycin addition can not further increase the expression of ER stress markers in IR, which may result from a maximal level of CHOP and Grp78 induced by IR.

Taken together, these results suggested that the beneficial effects of nicorandil are mainly due to the reduction of ER stress in the IR injury.

Effects of nicorandil on suppression of IR-induced ER stress is mediated by PI3k/Akt pathway

PI3K-Akt has been widely reported to be one of the most important survival pathways in a variety of cell types, and inactivation of PI3K-Akt is important for inducing CHOP expression in ER-stressed cells [32, 33]. To ask whether PI3K/Akt pathway was involved during nicorandil treatment, we examined the activity of PI3K-Akt pathway in IR heart. As shown in Figure 6A and 6B, a marked increase of AKT phosphorylation was observed in IR rat heart treated with nicorandil, compared with IR control heart, as determined by western blot.

We further incubated the IR hearts with specific PI3K/Akt inhibitor wortmannin. By blocking the PI3K/Akt signaling pathway, we found that nicorandil was unable to suppress the ER stress protein expression. The level of CHOP and Grp78 was comparable to that in the IR group without nicorandil treatment (Fig. 6A, 6C and 6D). Moreover, wortmannin abolished the effect of nicorandil on decreasing the expression of BAX level and increaseing the antiapoptotic protein BCL2 level during IR (Fig. 6A, 6E and 6F). Accordingly, the nocorandil 
Fig. 5. Tunicamycin blocks nicorandil-induced protection against IR. (A) Representative immunoblots (upper panel) and averaged densitometric results of immunoblotting for ER stress related apoptosis signaling protein markers GRP78 and CHOP (low panel). GAPDH was used as a loading control. (B to E) Effects of nicorandil on hemodynamic changes in the hearts subjected to IR with and without ER stress-inducing agent Tunicamycin. Nic PC: nicorandil preconditioning. Nicorandil (Nic, $100 \mathrm{mM}$ ) was added before ischemia for 5 min with a 5 -min washout before ischemia. Tunicamycin (TM, 2.5 $\mathrm{mg} / \mathrm{ml}$ ) was added before ischemia for 5 min with a 10-min washout before ischemia. $n=4$ each. Values are the Mean \pm S.E.M. ${ }^{*} \mathrm{P}<0.05,{ }^{* *} \mathrm{P}$ $<0.01$ compared to balance group. $\# \mathrm{P}<0.05$, \#\#P $<0.01$ compared to IR group. $\dagger \mathrm{P}<0.05, \uparrow+\mathrm{P}<0.01$ compared to $\mathrm{I} / \mathrm{R}$ hearts pretreated with nicorandil alone.

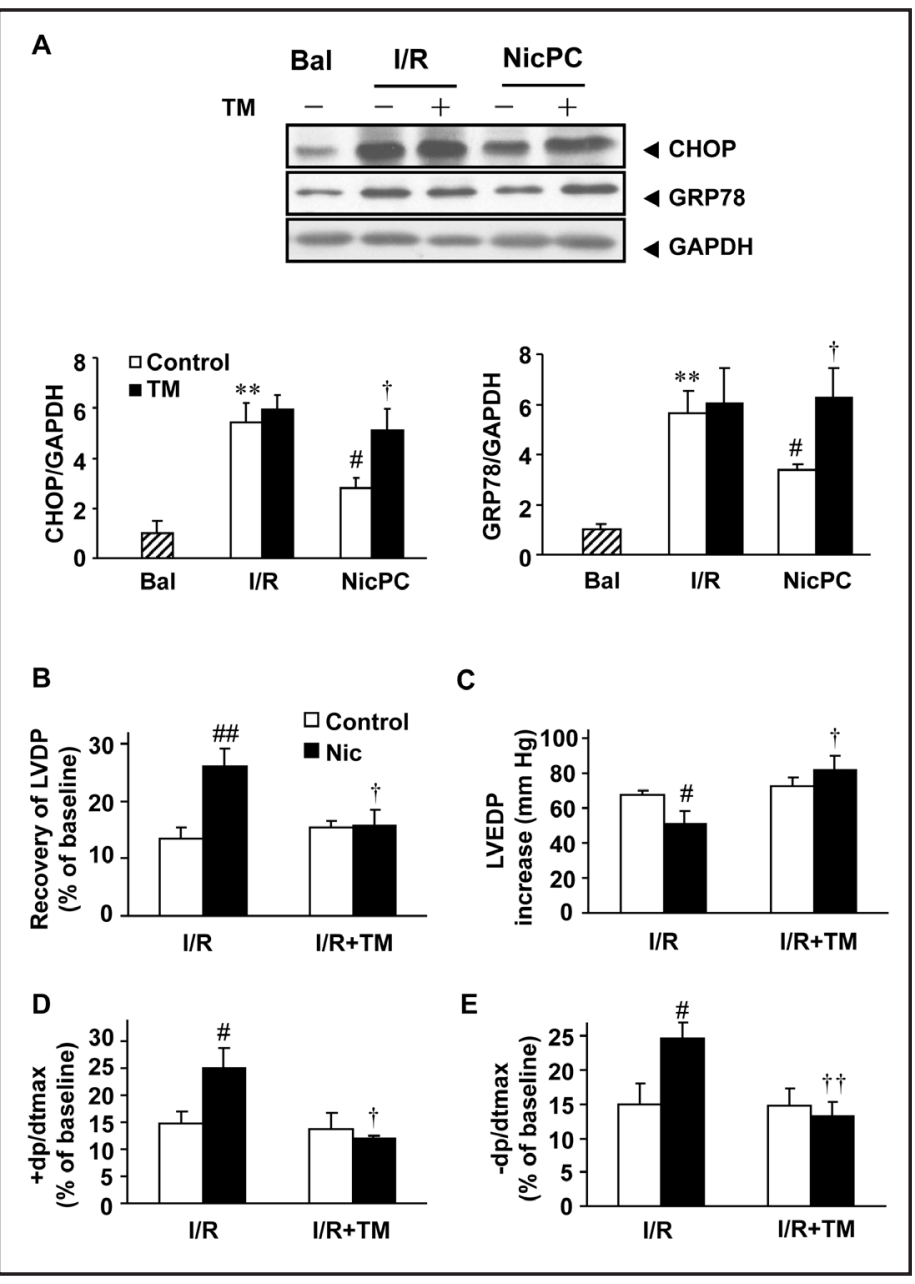

cardioprotection function was also blocked by inhibition of PI3K/Akt pathway (Fig. 6GJ). Furthermore, the infarct size was significant increased after treatment of wortmannin compared to the IR control (Fig. 7). Taken together, these results indicate that the protective effect of nicorandil on cardiac performance is dependent on PI3K/Akt signaling activity.

\section{Discussion}

Amounts of evidence have demonstrated that nicorandil, as a potassium channel opener and an nitric oxide (NO) donor, improves the recovery of the post-ischaemic contractile dysfunction of the heart and reduces the infarct size [24-26, 34]. Therefore, nicorandil is used in the treatment of angina and acute heart failure. However, the underlying mechanism of how nicorandil improves IR recovery is not fully understood. In this study, we clearly demonstrated that in rat IR model, pretreatment of nicorandil greatly induces cardioprotection via efficient activation of downstream anti-apoptotic targets. Furthermore, our results indicate that nicorandil ameliorates IR-induced mycardium damage by reducing ER stress and its function is dependent on the activation of PI3K/Akt pathway.

ER stress triggered apoptosis signaling includes the activation of CHOP and JNK signaling pathways, and consequently leads to IR injury [35, 36]. Our data confirmed that ER stress responses are involved in IR insults, and ER stress is essential for IR recovery. Through inhibiting ER stress by nicorandil, the IR recovery was significantly improved. Our results suggested that drugs targeting ER stress could be a promising strategy to cure IR. 
A

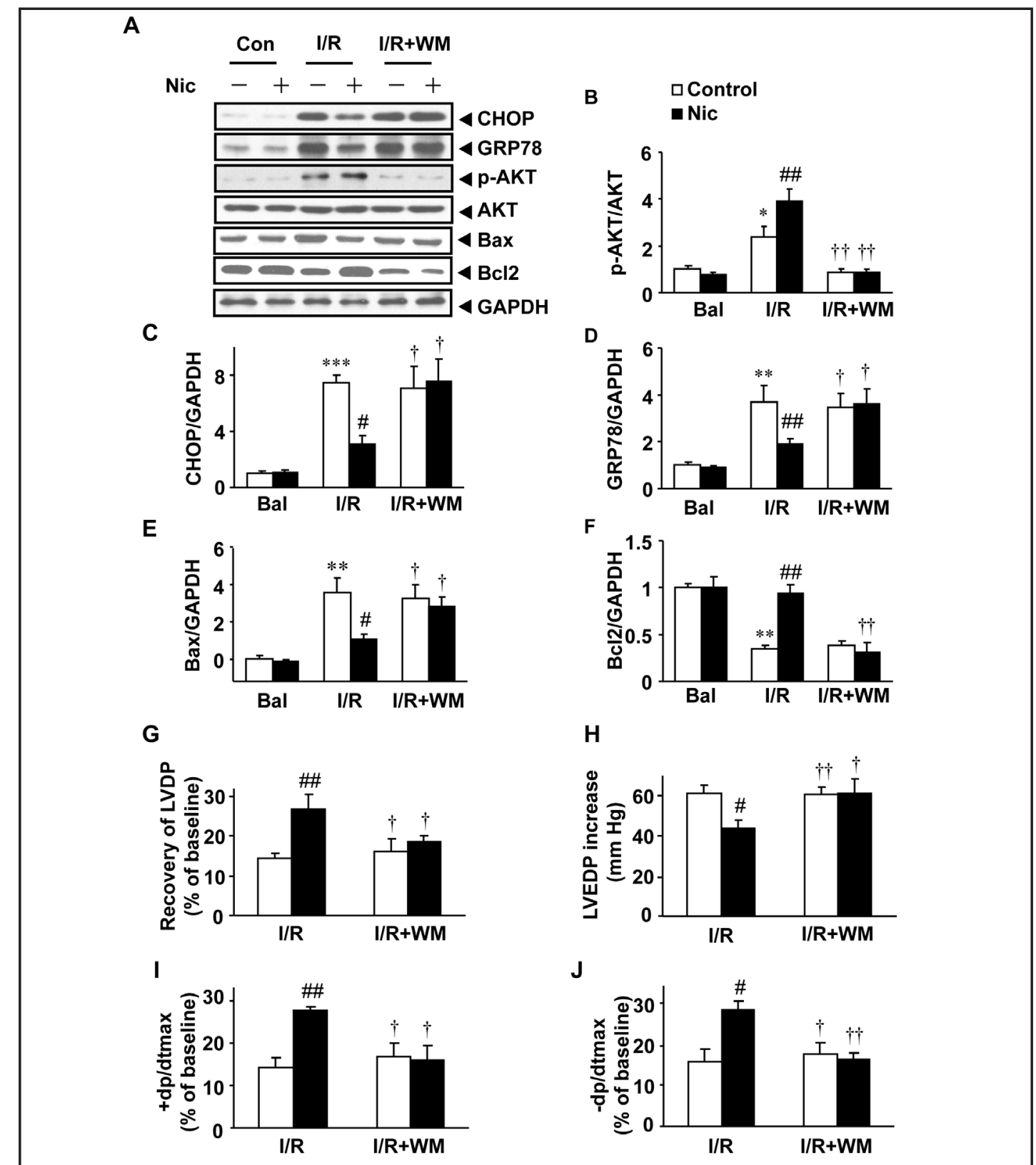

Fig. 6. The role of PI3K/Akt signaling in the cardioprotection by nicorandil was evaluated by using an Akt inhibitor wortmannin. (A-C) Representative immunoblots (A) and averaged densitometric results of immunoblotting for phos-Akt and total-Akt (B), GRP78 (C), CHOP (D), Bax (E) as well as Bcl-2 (F). GAPDH was used to demonstrate equal protein loading. (G-J) Effects of nicorandil on hemodynamic changes in the hearts subjected to IR with and without Akt inhibitor wortmannin. Nic PC: nicorandil preconditioning. Nicorandil (Nic, $100 \mathrm{mM}$ ) was added before ischemia for $5 \mathrm{~min}$ with a 5 -min washout before ischemia. Wortmannin (WM, $100 \mathrm{nM}$ ) was added before ischemia for $5 \mathrm{~min}$ with a 10-min washout before ischemia. $\mathrm{n}=4$ each. Values are the Mean \pm S.E.M. ${ }^{*} \mathrm{P}<0.05,{ }^{* *} \mathrm{P}<0.01,{ }^{* * *} \mathrm{P}<0.001$ compared to balance group. \# $\mathrm{P}<0.05$, \#\#P $<$ 0.01 compared to IR group. $\dagger \mathrm{P}<0.05, \dagger+\mathrm{P}<0.01$ compared to I/R hearts pretreated with nicorandil alone.

Nicorandil has been reported to exert its cardiopreotection role through activation of mitochondrial ATP-sensitive potassium $\left(\right.$ (mitoK $_{\text {ATP }}$ ) channels in several experimental animal models of myocardial IR $[26,37,38]$ However, whether nicorandil is associated with the ER response in the heart IR remains largely unknown. In neuronal cells, similar potassium channel openers protect neurons against apoptosis following cerebral IR injury by inhibition 


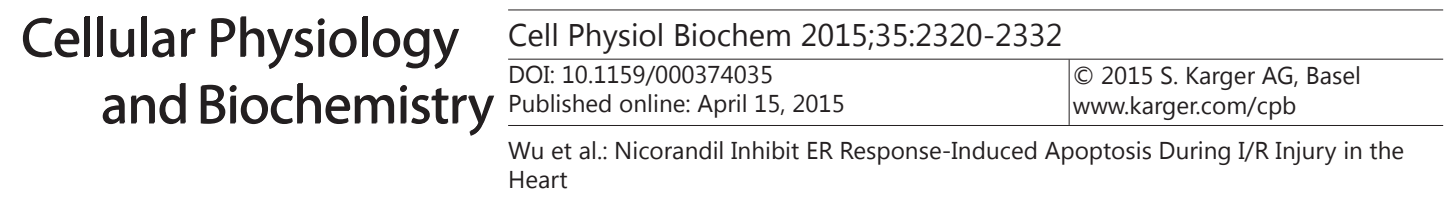

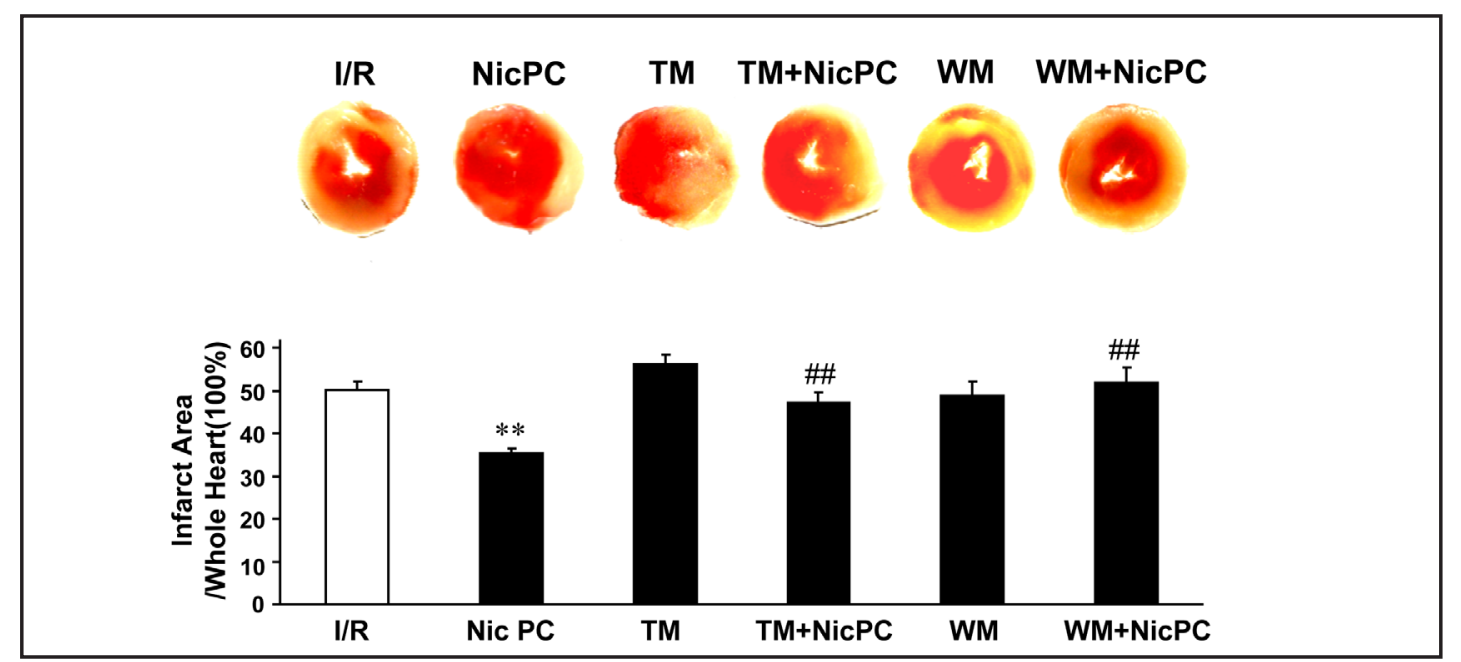

Fig. 7. Effects of nicorandil on infarct size in the hearts subjected to IR with and without ER stress inducer Tunicamycin and Akt inhibitor wortmannin. Nic PC: nicorandil preconditioning.Nicorandil (Nic, $100 \mathrm{mM}$ ) was added before ischemia for $5 \mathrm{~min}$ with a 5 -min washout before ischemia. Tunicamycin (TM, $2.5 \mathrm{mg} / \mathrm{ml}$ ) or wortmannin $(100 \mathrm{nM})$ was added before ischemia for $5 \mathrm{~min}$ with a 10-min washout before ischemia. $\mathrm{n}=$ 4 each. Values are the Mean \pm S.E.M. ${ }^{* *} \mathrm{P}<0.01$, compared to IR group; $\# \mathrm{P}<0.05$, \#\#P $<0.01$, \#\#\# $<0.001$ compared to I/R hearts pretreated with nicorandil alone.

of the ER stress pathway [39]. We showed here that the administration of nicorandil can actually suppressed endogenous increases of GRP78 and CHOP triggered by IR, and the cardioprotection of nicorandil was substantially abolished by ER-stress inducer. The finding is consitent with a recent report that nicorandil significantly reduces the expression levels of ER stress markers, CHOP and GRP78 in atherosclerotic lesions [40]. Therefore, our study domenstrated that nicorandil exerts its cardioprotection function via inhibiting ER stress during IR injury. Our study provides important mechanistic insights of cardioprotection attributed by nicorandil.

In deed, there are many ER stress pathways involved in IR. ER stress triggers the yinyang balance of cell survival versus cell death [41]. The cell reacts to ER stress by initiating a defensive process, called the unfolded protein response (UPR). UPR could either neutralize the stressful insult or adapt to it [4]. There are three signalling branches based on three types of ER transmembrane proteins: (i) inositol-requiring kinase/endoribonuclease 1 (IRE1), (ii) protein kinase activated by double-stranded RNA (PKR)-like ER kinase (PERK), and (iii) activating transcription factor 6 (ATF6) $[4,5]$. Like described in the introduction of our manuscript, GRP78, as a master regulator of ER stress, plays a key role in the cell's attempt to adapt and survive. GRP78 is bound to all these three ER transmembrane proteins [6, 7]. CHOP represents the later status of ER stress in which the apoptotic program is initiated [21, 22]. Both of which are shown to be essential to IR induced apoptosis. Therefore, in our study, we primarily focused on these two important ER stress markers. It would be interesting to determine the role of other ER stress pathway in nicorandil-treated IR response.

It is well established that PI3K/Akt signaling, as a key component of RISK pathway, is involved in cardiac protection [42-44]. The activation of endogenous Akt plays a critical role in controlling cell survival by resisting ER stress-induced cell death $[33,44]$. The direct link of the PI3K/Akt pathway with angina drugs would greatly facility our understanding of IR injury management. In the present study, we confirm that PI3K/Akt is involved in modulating ER stress during heart IR, and we also provided evidences that PI3K/Akt pathway is essential for nicorandil-mediated cadioprotection. Of note, there are many other surivival signaling pathways, such as JAK2/STAT3 [45]. Whether those signaling pathways were also involved in the cardiopreotection of nicorandil requires further investigation. Nevertherless, our study established the relationship between a specific drug and ER stress. It also expands the 


\section{Cellular Physiology Cell Physiol Biochem 2015;35:2320-2332 \begin{tabular}{l|l} 
Dol: 10.1159/000374035 & $\begin{array}{l}\text { 2015 S. Karger AG, Basel } \\
\text { www.karger.com/cpb }\end{array}$ \\
\cline { 2 - 3 }
\end{tabular} \\ Wu et al.: Nicorandil Inhibit ER Response-Induced Apoptosis During I/R Injury in the Heart}

knowledge of IR-induced cellular signaling and cast light to potential clinic implications of PI3K/Akt pathway.

In conclusion, we identified a clinically used anti-anginal drug, nicorandil, as a pharmacological ER stress inhibitor in IR. Since ER-stress is observed in a broad category of cardiovascular diseases including AMI. Our results indicated that nicorandil might also have potential clinical benefits not only in IR heart insults, but also in other pathological conditions of heart diseases in which ER-stress is also involved.

\section{Acknowledgements}

This study was supported by Natural Science Fundation of hubei province and Research Project of Hubei Provincial Department of Education (B20111201).

\section{Disclosure Statement}

The authors declare that there are no conflicts of interest.

\section{Reference}

1 Kalogeris T, Baines CP, Krenz M, Korthuis RJ: Cell biology of ischemia/reperfusion injury. Int Rev Cell Mol Biol 2012;298:229-317.

2 Eltzschig HK, Eckle T: Ischemia and reperfusion--from mechanism to translation. Nat Med 2011;17:13911401.

3 Thom T, Haase N, Rosamond W, Howard VJ, Rumsfeld J, Manolio T, Zheng ZJ, Flegal K, O'Donnell C, Kittner S, Lloyd-Jones D, Goff DC Jr, Hong Y, Adams R, Friday G, Furie K, Gorelick P, Kissela B, Marler J, Meigs J, Roger V, Sidney S, Sorlie P, Steinberger J, Wasserthiel-Smoller S, Wilson M, Wolf P, American Heart Association Statistics C, Stroke Statistics S: Heart disease and stroke statistics--2006 update: A report from the american heart association statistics committee and stroke statistics subcommittee. Circulation 2006;113:e85-151.

4 Schroder M, Kaufman RJ: Er stress and the unfolded protein response. Mutat Res 2005;569:29-63.

5 Xu C, Bailly-Maitre B, Reed JC: Endoplasmic reticulum stress: Cell life and death decisions. J Clin Invest 2005;115:2656-2664.

6 Hammadi M, Oulidi A, Gackiere F, Katsogiannou M, Slomianny C, Roudbaraki M, Dewailly E, Delcourt P, Lepage G, Lotteau S, Ducreux S, Prevarskaya N, Van Coppenolle F: Modulation of er stress and apoptosis by endoplasmic reticulum calcium leak via translocon during unfolded protein response: Involvement of grp78. FASEB J 2013;27:1600-1609.

7 Li N, Zoubeidi A, Beraldi E, Gleave ME: Grp78 regulates clusterin stability, retrotranslocation and mitochondrial localization under er stress in prostate cancer. Oncogene 2013;32:1933-1942.

8 Liu G, Shang Y, Yu Y: Induced endoplasmic reticulum (er) stress and binding of over-expressed er specific chaperone grp78/bip with dimerized epidermal growth factor receptor in mammalian cells exposed to low concentration of n-methyl-n'-nitro-n-nitrosoguanidine. Mutat Res 2006;596:12-21.

9 Lee AS: The er chaperone and signaling regulator grp78/bip as a monitor of endoplasmic reticulum stress. Methods 2005;35:373-381.

10 Rao RV, Peel A, Logvinova A, del Rio G, Hermel E, Yokota T, Goldsmith PC, Ellerby LM, Ellerby HM, Bredesen DE: Coupling endoplasmic reticulum stress to the cell death program: Role of the er chaperone grp78. FEBS Lett 2002;514:122-128.

11 Martindale JJ, Fernandez R, Thuerauf D, Whittaker R, Gude N, Sussman MA, Glembotski CC: Endoplasmic reticulum stress gene induction and protection from ischemia/reperfusion injury in the hearts of transgenic mice with a tamoxifen-regulated form of atf6. Circ Res 2006;98:1186-1193. 


\section{Cellular Physiology Cell Physiol Biochem 2015;35:2320-2332 \begin{tabular}{ll|l} 
and Biochemistry & Dublished online: April 15, 2015 & $\begin{array}{l}\text { C 2015 S. Karger AG, Basel } \\
\text { www.karger.com/cpb }\end{array}$ \\
\hline
\end{tabular} \\ Wu et al.: Nicorandil Inhibit ER Response-Induced Apoptosis During I/R Injury in the Heart}

12 Liu XH, Zhang ZY, Sun S, Wu XD: Ischemic postconditioning protects myocardium from ischemia/ reperfusion injury through attenuating endoplasmic reticulum stress. Shock 2008;30:422-427.

13 Vitadello M, Penzo D, Petronilli V, Michieli G, Gomirato S, Menabo R, Di Lisa F, Gorza L: Overexpression of the stress protein grp94 reduces cardiomyocyte necrosis due to calcium overload and simulated ischemia. FASEB J 2003;17:923-925.

14 Okada K, Minamino T, Tsukamoto Y, Liao Y, Tsukamoto O, Takashima S, Hirata A, Fujita M, Nagamachi Y, Nakatani T, Yutani C, Ozawa K, Ogawa S, Tomoike H, Hori M, Kitakaze M: Prolonged endoplasmic reticulum stress in hypertrophic and failing heart after aortic constriction: Possible contribution of endoplasmic reticulum stress to cardiac myocyte apoptosis. Circulation 2004;110:705-712.

15 Breckenridge DG, Germain M, Mathai JP, Nguyen M, Shore GC: Regulation of apoptosis by endoplasmic reticulum pathways. Oncogene 2003;22:8608-8618.

16 Glembotski CC: Endoplasmic reticulum stress in the heart. Circ Res 2007;101:975-984.

17 Rao J, Yue S, Fu Y, Zhu J, Wang X, Busuttil RW, Kupiec-Weglinski JW, Lu L, Zhai Y: Atf6 mediates a proinflammatory synergy between er stress and tlr activation in the pathogenesis of liver ischemia-reperfusion injury. Am J Transplant 2014;14:1552-1561.

18 Vekich JA, Belmont PJ, Thuerauf DJ, Glembotski CC: Protein disulfide isomerase-associated 6 is an atf6inducible er stress response protein that protects cardiac myocytes from ischemia/reperfusion-mediated cell death. J Mol Cell Cardiol 2012;53:259-267.

19 Szegezdi E, Duffy A, O'Mahoney ME, Logue SE, Mylotte LA, O'Brien T, Samali A: Er stress contributes to ischemia-induced cardiomyocyte apoptosis. Biochem Biophys Res Commun 2006;349:1406-1411.

20 Ron D, Walter P: Signal integration in the endoplasmic reticulum unfolded protein response. Nat Rev Mol Cell Biol 2007;8:519-529.

21 Miyazaki Y, Kaikita K, Endo M, Horio E, Miura M, Tsujita K, Hokimoto S, Yamamuro M, Iwawaki T, Gotoh T, Ogawa H, Oike Y: C/ebp homologous protein deficiency attenuates myocardial reperfusion injury by inhibiting myocardial apoptosis and inflammation. Arterioscler Thromb Vasc Biol 2011;31:1124-1132.

22 Groenendyk J, Sreenivasaiah PK, Kim do H, Agellon LB, Michalak M: Biology of endoplasmic reticulum stress in the heart. Circ Res 2010;107:1185-1197.

23 Frampton J, Buckley MM, Fitton A: Nicorandil. A review of its pharmacology and therapeutic efficacy in angina pectoris. Drugs 1992;44:625-655.

24 Kitakaze M, Asakura M, Kim J, Shintani Y, Asanuma H, Hamasaki T, Seguchi O, Myoishi M, Minamino T, Ohara T, Nagai Y, Nanto S, Watanabe K, Fukuzawa S, Hirayama A, Nakamura N, Kimura K, Fujii K, Ishihara M, Saito Y, Tomoike H, Kitamura S, investigators JW: Human atrial natriuretic peptide and nicorandil as adjuncts to reperfusion treatment for acute myocardial infarction ( $\mathrm{j}$-wind): Two randomised trials. Lancet 2007;370:1483-1493.

25 Ishii H, Ichimiya S, Kanashiro M, Amano T, Imai K, Murohara T, Matsubara T: Impact of a single intravenous administration of nicorandil before reperfusion in patients with st-segment-elevation myocardial infarction. Circulation 2005;112:1284-1288.

26 Akao M, Teshima Y, Marban E: Antiapoptotic effect of nicorandil mediated by mitochondrial atp-sensitive potassium channels in cultured cardiac myocytes. J Am Coll Cardiol 2002;40:803-810.

27 Schalla S, Higgins CB, Chujo M, Saeed M: Effect of potassium-channel opener therapy on reperfused infarction in hypertrophied hearts: Demonstration of preconditioning by using functional and contrastenhanced magnetic resonance imaging. J Cardiovasc Pharmacol Ther 2004;9:193-202.

28 Lindy S, Turto H, Uitto J, Helin P, Lorenzen I: Injury and repair in arterial tissue in the rabbit. Analysis of DNA, rna, hydroxyproline, and lactate dehydrogenase in experimental arteriosclerosis. Circ Res 1972;30:123-130.

29 Lee Y, Gustafsson AB: Role of apoptosis in cardiovascular disease. Apoptosis 2009;14:536-548.

30 McCullough KD, Martindale JL, Klotz LO, Aw TY, Holbrook NJ: Gadd153 sensitizes cells to endoplasmic reticulum stress by down-regulating bcl 2 and perturbing the cellular redox state. Mol Cell Biol 2001;21:1249-1259.

31 Kroemer G, Galluzzi L, Brenner C: Mitochondrial membrane permeabilization in cell death. Physiol Rev 2007;87:99-163.

32 Song G, Ouyang G, Bao S: The activation of akt/pkb signaling pathway and cell survival. J Cell Mol Med 2005;9:59-71. 


\section{Cellular Physiology Cell Physiol Biochem 2015;35:2320-2332

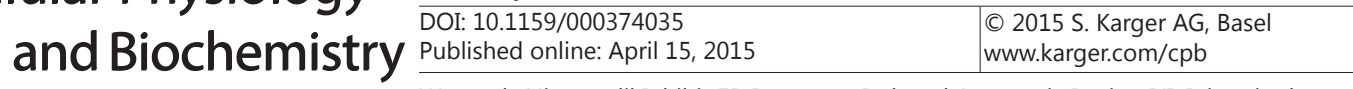 \\ Wu et al.: Nicorandil Inhibit ER Response-Induced Apoptosis During I/R Injury in the Heart}

33 Hyoda K, Hosoi T, Horie N, Okuma Y, Ozawa K, Nomura Y: Pi3k-akt inactivation induced chop expression in endoplasmic reticulum-stressed cells. Biochem Biophys Res Commun 2006;340:286-290.

34 Tang XL, Xuan YT, Zhu Y, Shirk G, Bolli R: Nicorandil induces late preconditioning against myocardial infarction in conscious rabbits. Am J Physiol Heart Circ Physiol 2004;286:H1273-1280.

35 Minamino T, Kitakaze M: Er stress in cardiovascular disease. J Mol Cell Cardiol 2010;48:1105-1110.

36 Thuerauf DJ, Marcinko M, Gude N, Rubio M, Sussman MA, Glembotski CC: Activation of the unfolded protein response in infarcted mouse heart and hypoxic cultured cardiac myocytes. Circ Res 2006;99:275-282.

37 Tsuchida A, Miura T, Tanno M, Sakamoto J, Miki T, Kuno A, Matsumoto T, Ohnuma Y, Ichikawa Y, Shimamoto $\mathrm{K}$ : Infarct size limitation by nicorandil: Roles of mitochondrial k(atp) channels, sarcolemmal k(atp) channels, and protein kinase c. J Am Coll Cardio 2002;40:1523-1530.

38 Mizumura T, Nithipatikom K, Gross GJ: Infarct size-reducing effect of nicorandil is mediated by the katp channel but not by its nitrate-like properties in dogs. Cardiovasc Res 1996;32:274-285.

39 Zhang H, Song LC, Jia CH, Lu YL: Effects of atp sensitive potassium channel opener on the mrna and protein expressions of caspase-12 after cerebral ischemia-reperfusion in rats. Neurosci Bull 2008;24:7-12.

40 Izumiya Y, Kojima S, Kojima S, Araki S, Usuku H, Matsubara J, Sakamoto K, Tsujita K, Nagayoshi Y, Kaikita K, Sugiyama S, Ogawa H: Long-term use of oral nicorandil stabilizes coronary plaque in patients with stable angina pectoris. Atherosclerosis 2011;214:415-421.

41 Schönthal AH: Endoplasmic reticulum stress: its role in disease and novel prospects for therapy. Scientifica 2012;2012:857516-857542.

42 Wolfrum S, Dendorfer A, Rikitake Y, Stalker TJ, Gong Y, Scalia R, Dominiak P, Liao JK: Inhibition of rho-kinase leads to rapid activation of phosphatidylinositol 3-kinase/protein kinase akt and cardiovascular protection. Arterioscler Thromb Vasc Biol 2004;24:1842-1847.

43 Bao W, Hu E, Tao L, Boyce R, Mirabile R, Thudium DT, Ma XL, Willette RN, Yue TL: Inhibition of rho-kinase protects the heart against ischemia/reperfusion injury. Cardiovasc Res 2004;61:548-558.

44 Hu P, Han Z, Couvillon AD, Exton JH: Critical role of endogenous akt/iaps and mek1/erk pathways in counteracting endoplasmic reticulum stress-induced cell death. J Biol Chem 2004;279:49420-49429.

45 Lecour S: Activation of the protective survivor activating factor enhancement (safe) pathway against reperfusion injury: Does it go beyond the risk pathway? J Mol Cell Cardiol 2009;47:32-40. 